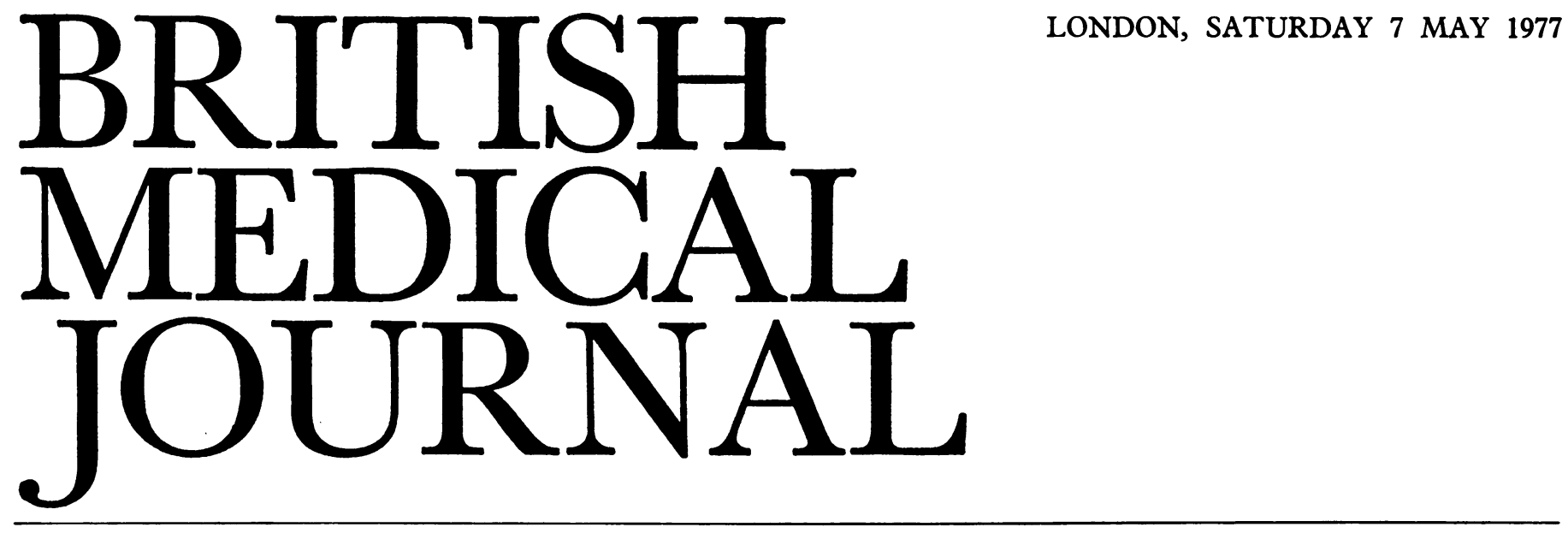

\title{
Medical management of endometriosis
}

Endometriosis is an enigmatic disease whose aetiology is uncertain. In consequence its treatment remains empirical, directed by known or presumed associations. For example, since endometriosis does not occur before the menarche nor usually remain active after the menopause, oestrogen is presumed to be essential for its development and continued activity. Doctors have used castration as a treatment for many years with excellent results, but (contrary to expectations) when women treated in this way are given oestrogen replacement therapy the disease is not usually reactivated. ${ }^{12} \mathrm{~A}$ further anomaly is the beneficial effect that pregnancy, a condition in which oestrogens are produced in large quantities, has upon the symptoms of endometriosis. Presumably the alleviating effect of pregnancy results from the suppression of menstruation.

Artificially induced amenorrhoea may be expected to relieve symptoms, and initially large doses of oestrogens were used for this purpose. The treatment proved unsatisfactory because of the cystic hyperplasia which occurred in the ectopic as well as the uterine endometrium. Though effective, androgens should not be used for long periods because of the risk of virilisation. Progestogens improve endometriosis both clinically and histologically by inducing a decidual reaction in the endometrium, the glandular elements of which undergo progressive inactivation and sometimes atrophy. Amenorrhoea induced by progestogens or by an oestrogen/progestogen combination given continuously for several months can give considerable relief, but cyclical or intermittent treatment, which does not result in amenorrhoea, is less effective. Pseudopregnancy after conservative surgery (in which the uterus is not removed) does not significantly reduce the incidence of subsequent operations for endometriosis, ${ }^{3}$ nor does it improve the pregnancy rate when compared with the results obtained from surgery alone. Furthermore, active endometriosis was found in 19 out of 21 patients having pelvic operations after pseudopregnancy, though some of these may have represented new rather than reactivated foci.

Regrettably, then, the beneficial effects of pseudopregnancy are often transient. When menstruation is resumed the ectopic endometrium becomes reactivated in most women if they are followed up for long enough. Of 17 patients $^{3}$ with endometriosis treated with an oestrogen/progestogen pseudopregnancy, symptoms recurred in 11 between two months and 14 years (average 5.5 years) after stopping hormone treatment. In five patients the recurrent symptoms were sufficiently severe to justify surgery.
The disappointing results of treatment by pseudopregnancy are probably due to the effects of the hormones available; their inherent oestrogenic or progestational properties have a direct stimulatory effect on the endometrium. An ideal drug should be able to suppress endogenous ovarian function, and so induce atrophy of the endometrium, while having no intrinsic oestrogenic or progestational properties of its own.

Danazol is a synthetic steroid derivative of ethisterone which, at least in part, meets these requirements. It is claimed to inhibit the synthesis or release of gonadotrophins from the pituitary. Greenblatt et $a l^{4}$ found that women lost their biphasic pattern of basal body temperature within four weeks of taking $800 \mathrm{mg}$ daily of danazol. Shortly afterwards their previously normal vaginal smears became hypoestrogenic, and within six to eight weeks atrophic changes appeared in the endometrium. Forty out of 42 patients studied developed amenorrhoea, and two continued to have scanty periods only. The characteristic midcycle surges of follicle stimulating hormone (FSH) and luteinising hormone ( $\mathrm{LH})$ disappeared during danazol therapy though basal gonadotrophin concentrations did not seem to be altered.

The effect of danazol-induced amenorrhoea on endometriosis was studied by Dmowski and Cohen ${ }^{5}$ in 39 patients, in all of whom the diagnosis had been confirmed by laparoscopy or laparotomy. All patients received $800 \mathrm{mg}$ of danazol daily for 3-11 months, and laparoscopy and endometrial biopsy were repeated on the last day of treatment. The extent of the endometriosis was recorded before and after treatment by endoscopic photography. All patients improved symptomatically. The second laparoscopy found no evidence of disease in 23 patients; scar tissue and haemosiderin deposits remained in 10; while histological evidence of inactive endometrial glands or stroma was found in six.

Equally impressive results were obtained by Friedlander ${ }^{6}$; on completion of treatment laparoscopy showed no evidence of endometriosis in 31 out of 39 patients and there was partial improvement in six. Friedlander commented that, to his surprise, pre-existing adhesions had disappeared in some of the patients, leaving the pelvic organs essentially normal. Pain was relieved in 21 of the 28 women in whom it had been the initial complaint and a further five said it was improved. Even more relevant was the observation by Greenblatt et $a l^{4}$ that nine patients with endometriosis whose symptoms had not improved with pseudopregnancy or with androgens did so when treated with danazol. The pregnancy rate after danazol treatment of infertile patients ${ }^{6} 7$ known to have endometriosis 
seems to be about $40 \%$ - comparable with the rate of $30-60 \%$ which may be expected after conventional treatment with pseudopregnancy or surgery.

Encouraging as the results of danazol treatment may be, there are no reports yet of the long-term follow-up of patients, and the benefits may prove short-lived. Ansbacher ${ }^{8}$ reported dramatic relief of symptoms in four patients treated with danazol, but their symptoms and signs of endometriosis recurred soon after stopping medication. Three of the four women underwent total hysterectomy and bilateral salpingooophorectomy within eight months of completing danazol treatment.

Despite the undoubted symptomatic relief which danazol provides, doubt has been cast recently on the claim that its primary effect is to suppress gonadotrophin production by the pituitary. Lind and $\mathrm{Cook}^{9}$ found normal plasma concentrations of FSH, LH, and oestradiol in three patients taking 600-1000 $\mathrm{mg}$ of danazol daily, though surprisingly they were unable to obtain endometrium at dilatation and curettage. Similarly Lieberman et $a^{10}$ found no change in plasma oestradiol, progesterone, basal $\mathrm{LH}$ and FSH concentrations, or response of $\mathrm{LH}$ to luteinising hormone releasing hormone (LHRH) after danazol treatment. However, they did find an impaired response of FSH to LHRH. Possibly danazol may act by directly inhibiting the effect of oestrogen on the endometrial cells. ${ }^{9}$ Whatever its mechanism of action, danazol may well prove valuable in treating endometriosis, so that it is unfortunate that a drug which shows such promise is so expensive in comparison with progestogen preparations: for unless the long-term results prove superior to alternative regimens it will be difficult to justify its routine use.

Medical treatment of endometriosis has a place in the management of endometriosis in women wishing to preserve their reproductive capacity and those with minimal, incompletely removed, or recurrent disease. Furthermore, it may be repeated without the disadvantage of recurrent laparotomies. It does not, however, replace surgery for definitive treatment of the disease, nor does it avoid the necessity for initial diagnostic laparoscopy.

1 Te Linde, R W, and Mattingley, R F, Operative Gynecology, p 219. Philadelphia, Lippincott, 1970.

2 Page, E W, Andrews, W C, and Larsen, G D, American fournal of Obstetrics and Gynecology, 1974, 118, 650.

${ }^{3}$ Andrews, W C, and Larsen, G D, American fournal of Obstetrics and Gynecology, 1974, 118, 643.

4 Greenblatt, R B, et al, Fertility and Sterility, 1971, 22, 102.

5 Dmowski, W P, and Cohen, M R, Obstetrics and Gynecology, 1975, 46, 147.

- Friedlander, R L, fournal of Reproductive Medicine, 1973, 10, 197.

7 Greenblatt, R B, Borenstein, R, and Hernandez-Ayup, S, American Fournal of Obstetrics and Gynecology, 1974, 118, 783.

8 Ansbacher, R, American fournal of Obstetrics and Gynecology, 1975, 121, 283.

- Lind, T, and Cook, D B, Lancet, 1976, 1, 1401.

10 Lieberman, B A, et al, British fournal of Obstetrics and Gynaecology, $1977,84,55$.

\section{Coronary artery spasm}

The concept that coronary arteries may go into spasm, provoking angina and even sudden death, is not new. The early arteriographic evidence of areas of transient coronary artery narrowing was commonly dismissed as an artefact produced by the dye injected into the vessel, and not until the careful observations of Sones and his colleagues ${ }^{1}$ were these radiographic abnormalities recognised to be clinically important.
Similarly, the failure to show structural coronary artery obstruction in patients dying from apparent ischaemic heart disease was initially attributed to either inaccurate clinical diagnosis or inadequate pathological technique. We now recognise, however, that myocardial infarction may, and frequently does, occur in patients with anatomically normal coronary arteries. ${ }^{2}$ Prinzmetal and his colleagues introduced the term "variant angina" for angina associated with ST segment elevation on the electrocardiogram, sometimes occurring without provocation in patients with a normal coronary arterial tree: "Temporary increased tonus"- -spasm in today's nomenclature -is thought to be the most likely cause. ${ }^{3-5}$

If spasm does occur, how may it arise? Experimental studies on animals show that the coronary arterial tree is responsive to a variety of stimuli, most of which are mediated through alpha-adrenoreceptors in the vessel wall. ${ }^{6}$ For example, stimulation of central sympathetic pathways or of the carotid sinus nerve or administration of noradrenaline ${ }^{7}$ or digitalis ${ }^{8}$ may provoke coronary vasoconstriction. Angina may be precipitated in susceptible patients by sudden exposure to the cold, and this may be studied experimentally using the cold pressor test, whereby one limb is plunged into ice cold water, so reflexly increasing vascular resistance. Braunwald and his colleagues ${ }^{9}$ recently examined the effect of this stimulus on coronary artery resistance in 24 patients with ischaemic heart disease (12 had virtually normal coronary vasculature on angiography, while the other 12 had obvious disease of the vessels). Coronary vascular resistance was calculated from measurements of coronary blood flow and mean arterial blood pressure. Mean blood pressure rose similarly on cold pressor testing in both groups, but in those patients with proved coronary vascular disease blood flow was depressed, while it was unchanged in the others.

The increase in coronary vascular resistance in the patients with vascular disease was compatible with vascular spasm; and angina was precipitated in four of the 12. These four showed a greater increase in coronary artery resistance than their fellows. To determine the role of alpha-adrenergic stimulation in these haemodynamic changes, the cold pressor test was repeated in three patients with coronary artery disease after intravenous administration of the alpha-adrenoreceptor blocking agent phentolamine. This prevented the change in coronary vascular resistance, lending further weight to the hypothesis that cold pressor stress produces changes in coronary vascular calibre mediated via the alpha-adrenergic receptors. Interestingly, spasm occurred only in those patients with pre-existing coronary vascular disease; patients with anginal symptoms but normal coronary vasculature were not affected.

Traditionally there is no better stimulus to the production of coronary vasodilatation than the products of an ischaemic myocardium itself. The therapeutic implication of this approach has been that even if potent coronary vasodilators are available their role is limited. Emphasis has been given to drugs such as glyceryl trinitrate, which lower total peripheral vascular resistance rather than affecting the coronary vessels specifically, and on beta-adrenoreceptor blocking agents, which act by relieving the cardiac work load. If coronary spasm is important, however, perhaps these concepts will have to be rethought, and the time may be ripe for a fresh study of alpha-adrenoreceptor blocking agents in angina, especially since beta-adrenoreceptor blocking agents may increase rather than decrease coronary artery tone.

If it is true, as suggested by Braunwald and his colleagues, ${ }^{9}$ that an increase in coronary vascular resistance is found only in 